\title{
Genetic diversity and population structure of Euscaphis japonica, a monotypic species
}

\author{
Wei-Hong Sun $^{1,2,3}$, De-Qiang Chen ${ }^{1,2,3}$, Rebeca Carballar-Lejarazu ${ }^{4}$, Yi Yang ${ }^{1,2,3}$, Shuang Xiang ${ }^{1,2,3}$, Meng-Yuan \\ Qiu $^{1,2,3}$, Zou Shuang-quan ${ }^{\text {Corresp. 1,2,3 }}$ \\ ${ }^{1}$ Fujian Agriculture and Forestry University, College of Forestry, Fujian Agriculture and Forestry University, Fuzhou, Fujian, China \\ ${ }^{2}$ Fujian Agriculture and Forestry University, Fujian Colleges and Universities Engineering Research Institute of Conservation and Utilization of Natural \\ Bioresources, Fujian Agriculture and Forestry, Fuzhou, Fujian, China \\ 3 Fujian Agriculture and Forestry University, Key Laboratory of National Forestry and Grassland Administration for Orchid Conservation and Utilization at \\ College of Landscape Architecture, College of Landscape Architecture, Fujian Agriculture and Forestry, Fuzhou, Fujian, China \\ 4 Department of Microbiology and Molecular Genetics, University of California, Irvine, USA, California, Irvine, USA
}

Corresponding Author: Zou Shuang-quan

Email address: zou@fafu.edu.com

Background: Understanding plant genetic diversity is important for effective conservation and utilization of genetic resources. Euscaphis japonica (Thunb.) Dippel, is a monotypic species with high phenotypic diversity, narrow distribution, and small population size. In this study, we estimated the genetic diversity and population structure of $E$. japonica using nine natural populations and inter-simple sequence repeat (ISSR) markers. Our results could provide a theoretical reference for future conservation and utilization of $E$. japonica.Results: We obtained a total of 122 DNA bands, of which 121 (99.18\%) were polymorphic. The average number of effective alleles ( $\mathrm{Ne}=1.4975)$, Nei's gene diversity index $(H=0.3016)$, and Shannon's information index $(I=0.4630)$ revealed that $E$. japonica possessed a high level of genetic diversity. We observed that $E$. japonica consisted of both deciduous and evergreen populations. UPGMA tree showed that the evergreen and deciduous $E$. japonica form a sister group. There is little genetic differentiation among geographic populations based on STRUCTURE analysis. The Dice's similarity coefficient between the deciduous and evergreen populations was low, and the Fst value was high, indicating that these two types of groups have high degree of differentiation. Conclusion: Rich genetic diversity has been found in $E$. japonica, deciduous $E$. japonica and evergreen $E$. japonica populations, and genetic variation mainly exists within the population. The low-frequency gene exchange between deciduous and evergreen populations may be the result of the differentiation of deciduous and evergreen populations. We suggest that in-situ protection, seed collection, and vegetative propagation could be the methods for maintenance and conservation of $E$. japonica populations. 


\section{Genetic diversity and population structure of Euscaphis}

\section{2 japonica, a monotypic species}

3

4 Sun Weihong 1,2,3, Chen De-Qiang 1,2,3, Rebeca Carballar-Lejarazú ${ }^{4}$, Yang Yi ${ }^{1,2,3}$, Xiang Shuang

5 1,2,3, Qiu Meng-Yuan ${ }^{1,2,3}$ and Zou Shuang-Quan 1,2,3*

6

$7 \quad{ }^{1}$ College of Forestry, Fujian Agriculture and Forestry University, Fuzhou, China

$8 \quad{ }^{2}$ Fujian Colleges and Universities Engineering Research Institute of Conservation and

9 Utilization of Natural Bioresources, Fujian Agriculture and Forestry University, Fuzhou, China

$10 \quad{ }^{3}$ Key Laboratory of National Forestry and Grassland Administration for Orchid Conservation

11 and Utilization at College of Landscape Architecture, College of Landscape Architecture, Fujian

12 Agriculture and Forestry University, Fuzhou, China

$13{ }^{4}$ Department of Microbiology and Molecular Genetics, University of California, Irvine, CA,

14 USA

15

16 Corresponding Author:

17 Zou Shuang-Quan 1,2,3*

18 Fuzhou, Fujian, China

19 Email address: zou@fafu.edu.cn 
21 Abstract

Background: Understanding plant genetic diversity is important for effective conservation and utilization of genetic resources. Euscaphis japonica (Thunb.) Dippel, is a monotypic species with high phenotypic diversity, narrow distribution, and small population size. In this study, we estimated the genetic diversity and population structure of E. japonica using nine natural populations and inter-simple sequence repeat (ISSR) markers. Our results could provide a theoretical reference for future conservation and utilization of E. japonica.

Results: We obtained a total of 122 DNA bands, of which $121(99.18 \%)$ were polymorphic. The average number of effective alleles $(N e=1.4975)$, Nei's gene diversity index $(H=0.3016)$, and Shannon's information index $(I=0.4630)$ revealed that E. japonica possessed a high level of genetic diversity. We observed that E. japonica consisted of both deciduous and evergreen populations. UPGMA tree showed that the evergreen and deciduous E. japonica form a sister group. There is little genetic differentiation among geographic populations based on STRUCTURE analysis. The Dice's similarity coefficient between the deciduous and evergreen populations was low, and the Fst value was high, indicating that these two types of groups have high degree of differentiation.

Conclusion: Rich genetic diversity has been found in E. japonica, deciduous E. japonica and evergreen E. japonica populations, and genetic variation mainly exists within the population. The low-frequency gene exchange between deciduous and evergreen populations may be the result of the differentiation of deciduous and evergreen populations. We suggest that in-situ protection, seed collection, and vegetative propagation could be the methods for maintenance and conservation of E. japonica populations.

\section{Introduction}

The genetic diversity of woody species is of great significance to their survival and persistence (Soejima et al., 2002). Woody species with high genetic diversity could hold a greater adaptive capacity and are able to adapt to survive in changing environments and under poor conditions (Gaafar et al., 2014; Eriksson et al., 1995; Hedrick et al., 2004). The fecundity and gene dispersal of woody species have been observed to shape their genetic diversity patterns (Mitchell-Olds et al., 2007). Species with weak regenerating abilities have lower genetic variation and less adaptive flexibility (Wang et al., 2011). Habitat fragmentation has an important impact on the demographic and genetic aspects of plant populations. Habitat fragmentation aggravates genetic erosion, and eventually leads to decreased individual fitness, 
54

thereby inhibiting population persistence (Travis et al., 2010; Yang et al., 2010). Besides, the loss of allele richness caused by decreased heterozygosity may reduce the opportunities for future adaptation of the population (Travis et al., 2010). Geographic range is also a contributing factor to a species' level of genetic diversity, as widely distributed species usually exhibit a higher level of diversity, while endemic woody species often show lower genetic diversity (Wei et al., 2012; Allly et al., 2000; Luan et al., 2006). Therefore, having a basic understanding of a species' genetic variation is key to developing effective conservation strategies, especially for endangered species or species with a small population (Booy et al., 200; Vicente et al., 2011).

Euscaphis japonica (Thunb.) Dippel, belongs to the Staphyleaceae family, is monotypic, and only distributed in southern China, Japan, and Korea (Li et al., 2008; Cheng et al., 2010). According to Flora of China records, E. japonica is a deciduous tree or shrub with odd-pinnate leaves, papery leaflets, sparsely serrulate margins with glandular teeth, and a soft, red, leathery pericarp with irregular ribs (Li et al., 2008). Previous studies have found that E. japonica exhibits significant phenotypic differences at different altitudes, and it can be classified as deciduous or evergreen based on its phenotypic markers (Sun et al., 2019). The deciduous $E$. japonica was characterized by its papery leaflets, serrulate margins, and prominent epidermis ribs, and the evergreen E. japonica was characterized by its membranous leaflets with obtuse, serrate margins, and its inconspicuous fruit epidermis rib (Sun et al., 2019). However, evaluating genetic variations across different populations using morphological characters is difficult because plant morphology varies under different growing conditions (Wang et al., 2005).

Furthermore, E. japonica is an excellent ornamental tree species for its butterfly-shaped fruit and red pericarp, and is currently cultivated on a large scale as an ornamental and medicinal tree species in Jiangxi and Fuzhou in China. It is also an important medicinal material in China since ancient times for the treatment of colds and coughs (Liang et al., 2019; Sun et al., 2019). Due to human interference and unreasonable picking of fruits, leaves, and branches for medicinal materials, some habitats of the natural E. japonica population have been largely fragmented. Whereas, there is short of a related research on E. japonica. A detailed study of its genetic diversity is therefore necessary to develop a strategy that can manage and maintain resilient, productive, and sustainable E. japonica forests (Iddrisu et al., 2005).

DNA-based molecular markers are not affected by environmental or physiological factors, making them suitable for estimating genetic diversity across plant species and populations (Mei et al., 2017; Tanya et al., 2011; Noormohammadi et al., 2013; Kaya, 2015). Inter-simple sequence repeat (ISSR) marker is a type of molecular markers based on inter-tandem repeats of short DNA sequences, which can determine intramolecular and intergenomic diversity by simultaneously revealing variations in unique regions of several loci in the genome. Because they provide simple, quick, efficient, and reproducible markers that can detect high levels of 
90 polymorphism (Reddy et al., 2002; Rakoczy-Trojanowska \& Bolibok, 2004), ISSR markers have

91 been used to analyze the genetic diversity of germplasm collections and to identify genotypes in 92 studies on wild hawthorn (Sheng et al., 2017), Magnolia wufengensis (Cheng et al., 2014), 93 Bergenia ciliata (Tiwari et al., 2015), and Sindora glabra (Yang et al., 2016). In this study, we collected samples from 83 E. japonica individuals across nine populations. We analyzed the genetic variation and population structure of E. japonica populations using ISSR markers. We aimed to determine the genetic variations within E. japonica populations and across different populations, reveal E. japonica's genetic structure, and recommend future management and conservation strategies.

\section{Materials \& Methods}

101

\section{Plant material}

Based on the samples collected from the previous phenotypic diversity of E. japonica (Sun et al., 2019), we have expanded the scope of field investigation and collected more samples from August 2016 to March 2017. During field observation, we found that E. japonica had two major categories, deciduous and evergreen, and we sampled these two types separately. All observed individuals were sampled, and the latitude, longitude, altitude, and specific habitats were recorded. We collected 83 samples across nine populations in Wuyi Mountain (WYC1, WYC2, WYC3, WYC4, and WYL), Taimu Mountain (TML), Daiyun Mountain (DYC and DYL), and Xishui National Nature Reserve (ZYL) (Fig. 1). The Wuyi Mountain range is about 550 kilometers long from the northeast to southwest, so five populations were collected. We collected and dried 10 to 15 fresh leaves from each sample using silica gel in sealed bags (Chase et al., 1991). The dry leaves were stored at $4^{\circ} \mathrm{C}$ in a refrigerator to be used for ISSR analysis.

The characteristics of the different population localities and morphologies are shown in Table 1.

\section{DNA isolation and purification}

We extracted genomic DNA from the E. japonica leaves using a modified acetyl trimethyl ammonium bromide method (Uddin et al., 2014). Fresh leaves (50 mg) without veins were ground into a fine powder using liquid nitrogen and a mortar. We then quickly transferred the powder to 2-mL microcentrifuge tubes with $700 \mu \mathrm{L}$ of extraction buffer $(1.4 \mathrm{M} \mathrm{NaCl}, 100 \mathrm{mM}$ Tris $\mathrm{HCl}$ (pH 8.0), $20 \mathrm{mM}$ EDTA, 1\% PVP, and 1\% $\beta$-mercaptoethanol) that had been preheated at $65^{\circ} \mathrm{C}$. This mixture was incubated at $65^{\circ} \mathrm{C}$ for $40 \mathrm{~min}$ with gentle shaking. After cooling for 10 min, we added chloroform: isopentyl alcohol mixture (24:1, equal volume: $700 \mu \mathrm{L})$ and centrifuged it at 12,000 rpm for $10 \mathrm{~min}$. The supernatant was transferred into a clean $2-\mathrm{mL}$ microcentrifuge tube, and two volumes of absolute ethanol were added and gently mixed using 
124 inversion. The DNA pellet was washed twice with 70\% (v/v) ethanol and dissolved in TE buffer

125

126

127

128

129

130

131

132

133

134

135

136

137

138

139

140

141

142

143

144

145

146

147

148

149

150

151

152

153

154

155

156

157

158

159 (pH 8.0) after air drying. We removed RNA contamination by adding $2 \mu \mathrm{L}$ of RNase (10 $\mathrm{mg} / \mathrm{mL}$ ) to each tube, incubated the tubes for $30 \mathrm{~min}$ at $37^{\circ} \mathrm{C}$, and then added $400 \mu \mathrm{L}$ of chloroform: isopentyl alcohol (24:1). Samples were centrifuged at $12,000 \mathrm{rpm}$ for $10 \mathrm{~min}$ at $4^{\circ} \mathrm{C}$ and the aqueous layer was transferred into a new tube. We added two volumes of chilled ethanol and $1 \% \mathrm{NaAc}(3 \mathrm{~mol} / \mathrm{L}, \mathrm{pH} 5.2)$ to precipitate the DNA. The DNA was washed with $70 \%$ ethanol and dissolved in $100 \mu \mathrm{L}$ of TE buffer after air drying. Each sample was diluted to 80 $\mathrm{ng} / \mathrm{mL}$ using TE buffer and was stored at $4^{\circ} \mathrm{C}$ for further ISSR analysis.

We measured the concentration of recovered DNA using NanoDrop ${ }^{\mathrm{TM}}$ spectrophotometry (Thermo Fisher Scientific, Waltham, MA, USA) at $260 \mathrm{~nm}$ and $280 \mathrm{~nm}$, and verified the purity of the DNA using $0.8 \%(\mathrm{w} / \mathrm{v})$ agarose gel. SYNGENE (BioRad, Hercules, CA, USA) was used to visualize and photograph the gel under a UV transilluminator.

\section{ISSR amplification}

PCRs contained $1 \mu \mathrm{L}$ of $80 \mathrm{ng}$ template DNA, $1 \mu \mathrm{L}$ of $0.5 \mu \mathrm{mol} / \mathrm{L}$ primer, $0.4 \mu \mathrm{L}$ of 0.2 $\mu \mathrm{mol} / \mathrm{L} \mathrm{dNTP}, 2 \mu \mathrm{L}$ of $2.5 \mathrm{mmol} / \mathrm{L} \mathrm{MgCl} 2,0.3 \mu \mathrm{L}$ of $1.5 \mathrm{U}$ Taq enzyme, $2 \mu \mathrm{L}$ of $10 \times$ PCR buffer, and sterile distilled water, for a final volume of $20 \mu \mathrm{L}$. ISSR primers were synthesized by Sangon Bioengineering (Shanghai, China) and all reagents were purchased from Solarbio (Beijing, China). We conducted PCR amplification using a Veriti ${ }^{\mathrm{TM}}$ 96-Well Thermal Cycler (Applied Biosystems, Foster City, CA, USA) as follows: initial denaturation at $95^{\circ} \mathrm{C}$ for $5 \mathrm{~min}$, 35 cycles at $94^{\circ} \mathrm{C}$ for $1 \mathrm{~min}$, at $55^{\circ} \mathrm{C}$ for $30 \mathrm{sec}$, and $72^{\circ} \mathrm{C}$ for $1 \mathrm{~min}$; final extension at $72^{\circ} \mathrm{C}$ for 7 min, and the tubes were subsequently maintained at $4^{\circ} \mathrm{C}$ before analysis. All ISSR primers had been initially tested, and 12 primers amplified DNA with polymorphic bands (Table 2). PCR products were analyzed on a $1.2 \%(\mathrm{w} / \mathrm{v})$ agarose gel in $1 \mathrm{x}$ TBE buffer and were visualized using the Gel Doc system (BioRad).

\section{Statistical analysis}

We scored amplification based on the amplicon bands from the gel photographs. The presence of bands at an amplicon level was scored as 1 and the absence was scored as 0 , which we used to calculate the raw data of the 0-1 matrix (Uddin et al., 2014). We used POPGENE 1.3.1 to analyze various genetic parameters, such as the number of alleles $(\mathrm{Na})$, the number of effective alleles $(\mathrm{Ne})$, Nei's genetic diversity $(H)$, Shannon's information index $(I)$, the percentage of polymorphic loci $(P P B)$, the level of gene flow $(\mathrm{Nm})$, and the Dice's similarity coefficient (GS) (Yeh et al., 1997). We calculated polymorphism information content (PIC) using the following formula: $\mathrm{PIC}=2 \mathrm{Pi}(1-\mathrm{Pi})$, where $\mathrm{Pi}$ is the frequency of polymorphic band occurrence in different primers (Khaleghi et al., 2017). The unweighted pair group method with the arithmetic average (UPGMA) and NTSYS 2.1 were used for dendrogram construction. To verify the UPGMA clustering results, we performed a cophenetic correlation analysis using NTSYS 2.1. 
160 Principal component analysis (PCA) was performed to show the E. japonica multiple

161 dimensional distributions in a scatter plot (NTSYS 2.1) (Wang et al., 2009). We used GenAlex

1626.5 to calculate the variance components within and between populations (Peakall et al., 2012).

163 The genetic structure of the E. japonica population was determined using STRUCTURE 2.3.1

164 based on Bayesian model-based clustering (Pritchard et al., 2000). The procedure was carried out

165 by selecting the correlated allele frequencies among populations and the admixture model. The 166 presumed populations $(K)$ denoted from 1 to 13 and estimated 20 independent runs for each $K$.

167 The operating parameter was a burn-in period of 100,000 and 100,000 Markov Chain Monte

168 Carlo replicates after burn-in. The optimal number of clusters was identified using Structure

169 Harvester (http://taylor0.biology.ucla.edu/struct_harvest/) (Earl \& vonHoldt, 2012).

170

171

172

173

174

175

176

177

178

179

180

181

182

183

184

185

186

187

188

189

190

191

192

193

\section{Results}

\section{ISSR polymorphism and genetic diversity}

We amplified a total of 122 identifiable bands, 121 of which were polymorphic bands. The polymorphic ratio $(P P B)$ was $99.18 \%$. The size of the PCR products ranged from 250 to 2,000 bp. The largest number of bands (14) was obtained using the UBC 808 primer, while only six bands were obtained using the UBC 890 primer. The highest PIC value was 0.62 (UBC 809), the lowest $P I C$ value was 0.48 (UBC 856), and the mean PIC per primer was 0.56 (Table 2). The high $P P B$ and $P I C$ values suggest that $E$. japonica has abounding genetic information, and that ISSR markers are appropriate to use in the genetic diversity analysis of E. japonica populations

Table 3 shows the statistical analysis of the E. japonica genetic parameters when using GenAIEx 6.5. At the species level, the average $N e$ was $1.4974, H$ was 0.3016 , and $I$ was 0.4630 . At the population level, the ranges of $H$ and $I$ across different populations were $0.0994-0.3688$ and $0.1594-0.3971$, respectively. Five populations had $H$ values greater than 0.2 : WYC2 (0.2607), WYC3 (0.2512), WYL (0.2360), DYL (0.2301), and WYC4 (0.2141). Four populations had $I$ values greater than 0.3: WYC2 (0.3971), WYC3 (0.3751), ZYL (0.3548), and DYL (0.3471). The five populations with the largest $N e$ values were WYC2 (1.4090), WYC3 (1.4276), ZYL (1.4034), DYL (1.3857), and WYC4 (1.3610). The five populations with the largest population polymorphic rates $(P P B)$ were WYC (86.07\%), WYC4 (72.13\%), WYC3 (70.49\%), ZYL (68.85\%), and DYL (67.21\%). Considering each genetic diversity index collectively, including the $H, I$, and $N e$ values, the WYC2 population had the highest genetic diversity, followed by WYC3 and WYC4.

\section{Genetic structure}

The UPGMA tree, based on individual samples, consisted of four major clusters. Most ZYL 
194 population samples and all WYL samples were grouped into cluster A. The remaining ZYL

195

196 samples, all TML samples, and DYL samples were grouped into cluster B. Cluster C contained WYC3 and WYC4 samples. Cluster D included WYC1, WYC2, and DYC samples. Clusters A and B consisted of deciduous E. japonica, and clusters C and D consisted of evergreen $E$. japonica (Fig. 2a). We based the PCA on genetic similarities to determine the genetic relationships between E. japonica populations and to create a two-dimensional display of these relationships. The PCA of the cumulative data grouped the nine populations into clusters I, II, and III, where cluster I included deciduous E. japonica and clusters II and III included evergreen E. japonica. Cluster II contained WYC1, WYC2, and DYC samples, and cluster III included WYC3 and WYC4 samples (Fig. 2b). The STRUCTURE analysis results indicated that the number of optimal clusters was four (Figs. 2c and 2d) based on the maximum delta $K=4$. Therefore, we divided the populations into four clusters (Fig. 2e): ZYL, TML, WYL, and DYL (purple); WYC2 (green); WYC1, DYC, and some WYC4 (blue); and the remaining WYC4 and WYC3 (yellow). The samples in the purple groups are deciduous E. japonica, and the samples in the green, blue, and yellow groups are evergreen E. japonica. Interestingly, the great isolation of population ZYL is not reflected in an unusual level of genetic distance (Figs. 2a and 2e). However, this population forms a distinct entity in the dendrogram. These results showed that the genetic differentiation between different geographic populations is not obvious. Furthermore, from Fig. 2e, there was little genetic infiltration between the evergreen and deciduous populations. The lower average gene flow $(\mathrm{Nm})$ of $E$. japonica $(\mathrm{Nm}=0.7804)$ also confirms the previous results (Table 4). In addition, we found that the deciduous $(\mathrm{Ne}=1.450, H=0.271, I=$ $0.413, P P B=84.43 \%)$ and evergreen $E$. japonica had rich genetic variation. $(N e=1.474, H=$ $0.290, I=0.446, P P B=97.54 \%$ ) (Table 3).

\section{Genetic differentiation}

The Dice's genetic similarity coefficient ranged from 0.729 to 0.938 . Two populations had relatively high Dice's similarity coefficients, indicating a close genetic relationship and small genetic differences between the two populations. The Dice's similarity coefficients were higher between WYC1 and WYC2 (0.954), WYC2 and WYC4 (0.921), WYC3 and WYC4 (0.936), and DYL and ZYL (0.938), while the Dice's similarity coefficients between DYC and TML (0.729), DYC and ZYL (0.793), WYL and TML (0.733), WYL and DHC (0.764), and WYL and WYC1 (0.766) were lower (Table 4). Genetic differentiation coefficient (Fst) values greater than 0.25 indicates greater genetic differentiation across populations (Wright, 1978). The Fst values for WYC1 and WYC2 (0.0883), WYC1 and WYC4 (0.2249), WYC2 and WYC4 (0.2070), WYC2 and WYC3 (0.2350), WYC3 and WYC4 (0.1509), and WYC3 and DYL (0.2172) populations were less than 0.25 , and the Fst values for other populations were all greater than 0.25 (Table 4). 
229

230

231

232

233

234

235

236

237

238

239

240

241

242

243

244

245

246

247

248

249

250

251

252

253

254

255

256

257

258

259

260

261

262

263

Overall, there was a high similarity coefficient and a low Fst value across deciduous or evergreen populations in the same mountain, and there was a lower similarity coefficient and high $F s t$ value between deciduous and evergreen populations.

The AMOVA analysis showed that $30 \%$ of total genetic variation existed across populations and $70 \%$ existed within populations (Table 5). The genetic variation within deciduous and evergreen populations accounted for $64 \%$ and $77 \%$ of the total variation, respectively. The genetic variation across different deciduous and evergreen populations accounted for $36 \%$ and $23 \%$ of the total variation, respectively. These results showed that for both deciduous E. japonica and evergreen E. japonica, most genetic variation was from within populations rather than from among populations.

\section{Discussion}

\section{Genetic diversity and differentiation}

We determined polymorphisms in nine E. japonica populations from China by estimating the genetic variability across different populations. Compared to previous reports, E. japonica's average $N e, H$, and $I$ values $(N e=1.4974, H=0.3016, I=0.4630)$ were higher than those observed in Staphylea bumalda (Staphylea, Staphyleaceae) $(N e=1.341, H=0.227, I=0.370)$ (Chen et al., 2014). These results indicate that E. japonica has high genetic variation at the species level. There are two types of E. japonica, deciduous and evergreen E. japonica. We evaluated the genetic diversity of E. japonica, deciduous E. japonica, and evergreen E. japonica, and found that they all have abundant genetic variation, and the genetic variation within the population was much greater than that between populations. Numerous previous studies have shown that most woody species have more variation within individual populations than across different populations (Hamrick et al., 1992; Wei et al., 2012). Woody species with long-lived, outcrossing breeding systems and animal seed dispersal have higher variations within populations than across populations (Albert et al., 2005; Schaal et al., 2010; Ramírez et al., 2014). E. japonica is a long-lived plant with a mixed mating system. Bee is the primary pollinator for E. japonica, meaning that pollen transfer can occur between adjacent populations (Sun et al., 2017), such as the WYC1 and WYC2 populations, WYC3 and WYC4 populations located in the Wuyi Mountain. The red pericarp of E. japonica attracts birds to eat the fruit that helps with seed dispersal. The lower interpopulation genetic diversity of E. japonica, deciduous E. japonica, and evergreen E. japonica and higher intrapopulation genetic diversity may be the results of its long life, mixed mating, and seed dispersal systems.

The lower Dice's_similarity coefficient and higher Fst value between the deciduous and evergreen populations confirmed that the deciduous and evergreen E. japonica experienced differentiation. Frequent gene flow can prevent genetic drift and reduce genetic differentiation 
264 (Tremblay et al., 2001). The STRUCTURE analysis and the lower average gene flow indicated

265

266

267 that there was less gene flow between the evergreen and deciduous populations. According to our long-term field observations, the florescence of deciduous E. japonica was observed from April to May, and the florescence of evergreen E. japonica was observed from May to June (Sun et al., 2017; Sun et al., 2019). Although bees are the primary pollinators, there is a small possibility of genetic interactions between the deciduous and evergreen populations in the same Mountain, such as DLY and DYC populations in Daiyun Mountain, and WYC1 and WYL populations in Wuyi Mountain. We speculate that little gene exchange between deciduous and evergreen E. japonica may be the result of the differentiation between these two type populations.

\section{Implications for $E$. japonica conservation}

The deciduous E. japonica is mainly distributed in southern China, Japan, Korea, and the evergreen E. japonica is distributed in Fujian, Jiangxi, Guangxi provinces in southern China ( $\mathrm{Li}$ et al., 2018; Sun et al., 2019). According to our field observations, the influence of human activities has been the main challenge affecting the survival of $E$. japonica populations (Sun et al., 2019). Therefore, based on the genetic diversity information of the population, an effective $E$. japonica conservation strategy is proposed to create favorable conditions for the innovation and effective utilization of E. japonica germplasm resources. We detected the highest genetic diversity in the WYC2 population $(P P B=86.07 \%, H=0.2607, I=0.3971)$, followed by WYC3 $(P P B=70.49 \%, H=0.2512, I=0.3751)$. The $\mathrm{WYC} 2$ and $\mathrm{ZYL}$ populations were located in the Jiangshi Nature Reserve and the Xishui Natural National Reserve, respectively, and the DYL population was located in sparsely populated natural villages. These habitats are well-preserved without frequent human interventions. Based on our UPGMA clustering, genetic diversity index, and population location, we suggest that the ZYL, DYL, WCY3, and WCY2 populations in clusters A, B, C, and D should be conserved in-situ. In-situ management of genetic resources can ensure that the majority of extant variation is preserved (Asddisalem et al., 2016; Negri et al., 2010).

Furthermore, we should work on recovering populations with low diversity, destroyed habitats, and small population sizes. TML and DYC habitats were shrinking due to human interventions. The WYC1 population is located near a city, and the WYC3 population was transplanted from local forests to forest farms because of habitat destruction. The populations with the lowest genetic diversity were WYL ( $P P B=20.49 \%, H=0.0803, I=0.1174)$ and DYC $(\mathrm{PPB}=21.31 \%, H=0.0738, I=0.1115)$ population. Therefore, we recommend an ex-situ conservation strategy of these populations. In ex-situ conservation, genetic resources are preserved outside their natural habitats in facilities such as seed banks and botanical gardens. These strategies aim to preserve genetic material in collections (Brush, 2000; Negri et al., 2010). 
300

301

302

303

304

305

306

307

308

\section{9}

310

311

312

313

314

315

316

317

318

319

320

321

322

323

324

325

326

327

328

329

330

331

332

333

Li et al. (2009) and Richards et al. (2010) confirmed that seed collections are a useful and effective way to maintain the size of most ex-situ populations, and that vegetative propagation can rapidly and effectively expand ex-situ population sizes (Li et al., 2018). In natural $E$. japonica populations, collecting and storing seeds are of significantly important, especially for populations with low genetic diversity and severely damaged habitats, such as TML, WYL, and WYC1. Other populations with very small individuals, such as DYC, can benefit from vegetative propagation and in situ conservation of genetic resources.

\section{Conclusions}

Our study showed that ISSR is a useful method for characterizing genetic diversity of $E$. japonica, which has high genetic diversity at the species level. UPGMA tree, PCA, and STRUCTURE analysis revealed that E. japonica can be divided into deciduous and evergreen $E$. japonica. AMOVA analysis indicated that intrapopulation genetic variation of E. japonica, deciduous E. japonica, and evergreen E. japonica was greater than genetic variation of interpopulation genetic variation, which may due to its mixed mating system and animal seed dispersal. The low average flow $(\mathrm{Nm})$ between deciduous and evergreen populations indicates that there was little genetic infiltration between this two types population, and structural analysis showed also confirmed this result. According to the similarity coefficient and Fst value, the deciduous and evergreen E. japonica may experience differentiation. We suggested that populations with high genetic diversity of which habitats that are less disturbed by human activities should be protected in-situ, and those populations with low genetic diversity, small populations, and whose habitats are disturbed by human activities should be protected by ex-situ, such as seed collection and vegetative propagation.

\section{Abbreviations}

ISSR: inter-simple sequence repeat marker; CTAB: acetyl trimethyl ammonium bromide; UPGMA: The unweighted pair group method with arithmetic average; N: north latitude; E: west longitude; A: altitude (m); No. of bands: the number of amplification band; PIC: the polymorphism information content; $\mathrm{Na}$ : the number of alleles; $\mathrm{Ne}$ : the number of effective alleles; $H$ : Nei's genetic diversity; $I$ : Shannon's information index; No. of polymorphic bands: the number of polymorphic bands; $P P B$ : the percentage of polymorphic loci; $\mathrm{Nm}$ : the level of gene flow; except $(H e)$ and unbiased excepted heterozygosity $(u H e)$. 


\section{Acknowledgments}

335 Special thanks to Bin Ou (College of Forestry, Jiangxi Environmental Engineering Vocational 336 College, Jiangxi Province), Zhu-gang Yi (Zunyi Forestry Bureau, Guizhou Province), Zhuang 337 Zou (Zhejiang Academy of Agricultural Sciences), and the Quangzhou Forestry Bureau, who 338 helped us find natural E. japonica populations.

339 
340

341

342

343

344

345

346

347

348

349

350

351

352

353

354

355

356

357

358

359

360

361

362

363

364

365

366

367

368

369

370

371

372

373

374

\section{References}

Albert T, Raspe O, Jacquemart AL, 2005. Diversity and spatial structure of clones in Vaccinium uliginosum populations. Can J Bot. 83(2):211-218.

Allly D, EIKassaby YA, Ritand K., 2000. Genetic diversity, differentiation and mating system in Mountain Hemlock (Tsuga Mertensiana) across british Columbia. Forest genetics. 7(2):97108.

Asddisalem AB, Bongers F, Kassahun T, Smulders MJM, 2016. Genetic diversity and differentiation of the frankincense tree (Boswellia papyrifera (Del.) Hochst) across Ethiopia and implications for its conservation. Forest Ecol Manag. 360(10):253-260.

Booy G, Hendriks RJJ, Smulders MJM, Groenendael JM, Vosman B, 2000. Genetic diversity and the survival of populations. Plant Biol. 2:379-395.

Brush SB, 2000. The issues of in situ conservation of crop genetic resources. $J$ Polym Sci Pol Phys. 44(14):1979-1984.

Castillo RFD, 1994. Factors influencing the genetic structure of Phacelia dubia, a species with a seed bank and large fluctuations in population size. Heredity. 71(71):446-458.

Chase MW, Hills HH, 1991. Silica Gel: An Ideal Material for Field Preservation of Leaf Samples for DNA Studies. Taxon. 40(2):215-220.

Chen L C, Wang Y, Wang YZ, 2014. ISSR primer selection and preliminary analysis of genetic diversity in natural populations of Staphylea bumalda. Journal of Anhui Agricultural University. 41(3):462-467.

Chen LY, Chen FJ, He SC, Ma LY, 2014. High genetic diversity and small genetic variation among populations of Magnolia wufengensis (Magnoliaceae), revealed by ISSR and SRAP markers. Electron J Biotechn. 17(6):268-274.

Cheng JJ, Zhang LJ, Cheng HL, Chiou CT, Lee IJ, Kuo YH, 2010. Cytotoxic Hexacyclic Triterpene Acids from Euscaphis japonica. J Nat Prod. 73(10):1655-1658.

Earl DA, vonHoldt BM, 2012. Structure Harvester: a website and program for visualizing Structure output and implementing the Evanno method. Conserv Genet Resour. 4:359-361. Eriksson G, Namkoong G, Roberds J, 1995. Dynamic conservation of forest tree gene resources. Forest Genetic Resources. 22(8):1738-1749.

Gaafar ARZ, AI-Qurainy F, Khan S, 2014. Assessment of genetic diversity in the endangered populations of Breonadia salicina (Rubiaceae) growing in the Kingdom of Saudi Arabia using inter-simple sequence repeat markers. BMC Genet. 15(1):1-10.

Hall P, Orrell LC, Bawa KS, 1994. Genetic diversity and mating system in a tropical tree, Carapa guianensis (Meliaceae). Am J Bot. 81(9):1104-1111.

Hamrick JL, 2004. Response of forest trees to global environmental changes. Forest Ecol Manag. 
197(1):323-335.

Hamrick JL, Godt MJW, Sherman-Broyles SL, 1992. Factors influencing levels of genetic diversity in woody plant species. New For. 6:95-124.

Harris A J, Chen PT, Xu XW, Zhang JQ, Yang X, Wen J, 2017. A molecular phylogeny of Staphyleaceae: Implications for generic delimitation and classical biogeographic disjunctions in the family. J Syst Evol. 55(2):1-18.

Hedrick PW. 2004. Recent developments in conservation genetics. For Ecol Manag. 97:3-19 Huang MX, Zou SQ, Chen L, 2013. Effect of appling artificial microbial agents on Euscaphis konishii seedings transplant and growth. Journal of Fujian College of Forestry. 33(1):25-27. Hayata, 1913. Euscaphis Konishii Hayata. Ic. Pl. Formos. 3:67.

Iddrisu MN, Ritland K, 2005. Genetic variation, population structure, and mating system in bigleaf maple (Acer macrophyllum Pursh). Can J Bot. 82(12):1817-1825.

Jump AS, Peñuelas J, 2006. Genetic effects of chronic habitat fragmentation in a wind-pollinated tree. Proceedings of the National Academy of Sciences of the United States of America. 103(21):8096-8100.

Kaya E, 2015. ISSR analysis for determination of genetic diversity and relationship in eight Turkish olive (Olea europaea L.) cultivars. Not Bot Hort Agrobo. 43(1):96-99.

Khaleghi E, Sorkheh K, Chaleshtori MH, Ercisli S, 2017. Elucidate genetic diversity and population structure of Olea europaea L. germplasm in Iran using AFLP and IRAP molecular markers. Biotech. 7(1):71.

Li BJ, Wang JY, Liu ZJ, Zhuang XY, Huang JX, 2018. Genetic diversity and ex situ conservation of Loropetalum subcordatum, an endangered species endemic to China. BMC Genet. 19(1):12.

Li DZ, Cai J, Wen J, 2008. Staphyleaceae. In: Wu ZY, Raven PH, Hong DY eds. Flora of China. Beijing: Science Press; St. Louis: Missouri Botanical Garden Press. 11:498-504

Li DZ, Pritchard HW, 2009. The science and economics of ex situ plant conservation. Trends Plant Sci. 14:614-621.

Luan S, Chiang T, Gong X. High Genetic Diversity vs, 2006. Low Genetic Differentiation in Nouelia insignis (Asteraceae), a narrowly distributed and endemic species in China, revealed by ISSR Fingerprinting. Ann Bot-London. 98(3):583-589.

Mei ZQ, Zhang XQ, Asaduzzaman Khan, Saber I, Liu XY, Zou CW, Fu JJ, 2017. Genetic analysis of Penthorum chinense Pursh by improved RAPD and ISSR in China. Electron J Biotechn. 30:6-11.

Meng LH, Yang R, Abbott RJ, Miehe G, Hu TH, Liu JQ, 2007. Mitochondrial and chloroplast phylogeography of Picea crassifolia Kom. (Pinaceae) in the Qinghai-Tibetan Plateau and adjacent highlands. Mol Ecol. 16:4128-4137. 
Mitchell-Olds T, Willis JH, Goldstein DB, 2007. Which evolutionary processes influence natural genetic variation for phenotypic traits? Nat Rev Genet. 8:845-856.

Monfared MA, Samsampour D, Sharifi-Sirchi G R, Sadeghi F, 2018. Assessment of genetic diversity in Salvadora persica, L. based on inter simple sequence repeat (ISSR) genetic marker. J Genet Engineer Biotechn. 16(2): 661-667.

Moslemi M, Zahravi M, Khaniki GB, 2010. Genetic diversity and population genetic structure of pomegranate (Punica granatum L.) in Iran using AFLP markers. Sci Hortic-Amsterdam. 126(4):441-447.

Negri V, Tiranti B, 2010. Effectiveness of in situ and ex situ conservation of crop diversity. What a Phaseolus vulgaris L. landrace case study can tell us. Genetica. 138(9-10):985-998.

Noormohammadi Z, Hasheminej-Adahangarani F Y, Sheidai M, Ghasemzadeh-Baraki S, Alishah O, 2013. Genetic diversity analysis in Opal cotton hybrids based on SSR, ISSR, and RAPD markers. Genet Mol Res. 12(1):256-269.

Rakoczy-Trojanowska M, Bolibok H, 2004. Characteristics and a comparison of three chasses of microsatellite-based markers and their application in plants. Cell Mol Biol Lett. 9:221-238.

Peakall R, Smouse PE, 2012. GenAlEx 6.5: genetic analysis in excel. Population genetic software for teaching and research-an update. Bioinformatics.28: 2537-9.

Petit RJ, Hampe A, 2006. Some Evolutionary Consequences of Being a Tree. Ann Rev Ecol Evol S. 37(1):187-214.

Pritchard JK, Stephens M, Donnelly P, 2000. Inference of population structure using multilocus genotype data. Genetics. 155:955.

Ramírez-Valiente JA, Valladares F, Aranda I, 2014. Exploring the impact of neutral evolution on intrapopulation genetic differentiation in functional traits in a long-lived plant. Tree Genet Genomes. 10(5):1181-1190.

Reddy MP, Sarla N, Siddiq EA, 2002. Inter simple sequence repeat (ISSR) polymorphism and its application in plant breeding. Euphytica. 128(1):9-17.

Richards CM, Lockwood DR, Volk GM, Walters C, 2010. Modelling demographics and genetic diversity in ex situ collections during seed storage and regeneration. Crop Sci. 50: 2440.

Schaal BA, Hayworth DA, Olsen KM, Rauscher JT, Smith WA, 2010. Phylogeographic studies in plants: problems and prospects. Mol Ecol. 7(4):465-474.

Sheng F, Chen SY, Tian J, Peng L, Xue Q, Lei W, Luo SP, Li J, 2017. Morphological and ISSR molecular markers reveal genetic diversity of wild hawthorns (Crataegus songorica K. Koch.) in Xinjiang, China. J Integr Agr. 16(11):2482-2495.

Sloan DB, Barr CM, Olson MS, Keller SV, Taylor DR, 2008. Evolutionary rate variation at multiple levels of biological organization in plant mitochondrial DNA. Mol Biol Evol. 25:243246. 
447

Soejima A, Maki M, Ueda K. 2002, Genetic variation in relic and isolated populations of Chionanthus retusus (Oleaceae) of Tsushima Island and the Tono region, Japan. Genes Genet Syst. 73(1):29-37.

Sun WH, Yuan XY, Liu ZJ, Lan SR, Tsai WC, Zou SQ, 2019. Multivariate analysis reveals phenotypic diversity of Euscaphis japonica population. PLoS ONE. 14(7):e0219046.

Sun WH, Yuan XY, Wu LJ, Xiang Shuang, Zou SQ, 2017. Stamen dynamics and breeding system of Euscaphis konishii Hayata. Plant Physiology Communications. 53(12):2215-2221.

Tanya P, Taeprayoon P, Hadkam Y, Srinives P, 2011. Genetic diversity among Jatropha and Jatropha-Related Species based on ISSR markers. Plant Mol Biol Rep. 29(1):252-264.

Tiwari V, Mahar KS, Singh N, Meena B, Nair KN, Datt B, Upreti DK, Tamta S, Rana TS, 2015. Genetic variability and population structure of Bergenia ciliata, (Saxifragaceae) in the Western Himalaya inferred from DAMD and ISSR markers. Biochem Syst Ecol. 60:165-170.

Tremblay RL, Ackerman JD, 2001. Gene flow and effective population size in Lepanthes (Orchidaceae): a case for genetic drift. Biol J Linn Soc Lond. 72(1):47-62.

Uddin MS, Sun W, He X, Silva JAT, Cheng Q, 2014. An improved method to extract DNA from mango Mangifera indica. Biologia. 69(2):133-138.

Vicente MJ, Segura F, Aguado M, Migliaro D, Franco JA, Martínez-Sánchez JJ, 2011. Genetic diversity of Astragalus nitidiflorus, a critically endangered endemic of SE Spain, and implications for its conservation. Biochem Syst Ecol. 39:175-182.

Vinceti B, Loo J, Gaisberger H, Zonneveld MJ, Schueler S, Konrad H, Kadu CAC, Geburek T, 2013. Conservation priorities for Prunus africana defined with the aid of spatial analysis of genetic data and climatic variables. PLoS ONE. 8 (3): 1-16.

Wang HZ, Wu ZX, Lu JJ, Shi NN, Zhao Y, Zhang ZT, Liu JJ, 2009. Molecular diversity and relationships among Cymbidium goeringii cultivars based on inter-simple sequence repeat (ISSR) markers. Genetica. 136(3):391-399.

Wang J, Li Z, Guo Q, Ren G, Wu Y, 2011. Genetic variation within and between populations of a desert poplar (Populus euphratica) revealed by SSR markers. Ann For Sci. 68(6):11431149.

Wei Z, Du Q, Zhang J, Li B, Zhang D, 2012. Genetic Diversity and Population Structure in Chinese Indigenous Poplar (Populus simonii) Populations Using Microsatellite Markers. Plant Mol Biol Rep. 31(3):620-632.

Wright S, 1978. Variability within and among natural populations. The University of Chicago Press, Chicago.

Yang JC, Li QQ, Yu N, Yin GT, Wu ZF, Li RS, Zou WT, 2016. Genetic diversity and structure among natural populations of Sindora glabra in Hainan Island, China as revealed by ISSR markers. Biochem Syst Ecol. 69:145-151. 
483 Yeh FC, Yang RC, Boyle T, Ye ZH, Mao JX, 1997. POPGENE, the user friendly shareware for 484 population genetic analysis. Molecular Biology and Biotechnology Centre. University of 485 Alberta, Edmonton, Canada.

486 Zou XX, Lu Y, Zou SQ, 2016. Preliminary test for Chemical Components from Leaves and 487 Branches of Euscaphis konishii Hayata. Chinese Wild Plant Resources. 35(1):70-72. 
488 Figure 1. Map showing the location of the sampled $E$. japonica populations.

489 The triangle represents a deciduous E. japonica population, and the circle or diamond represents 490 an evergreen E. japonica population.

491

Figure 2. E. japonica population genetic structure.

(a) ISSR dendrogram of E. japonica samples. (b) Two-dimensional picture of principal component analysis (PCA) using the ISSR genetic similarity matrix of all E. japonica samples. The plot was generated using a Dice's similarity matrix and NTSYS-pc software. The $\mathrm{x}$ and $\mathrm{y}-$ axis were converted from the Dice's genetic similarity matrix of E. japonica populations using NTSYS. The same color means that the sample came from the same population. Each triangle represents a deciduous E. japonica sample, and each circle or diamond represents an evergreen E. japonica sample. (c) Estimated mean logarithmic likelihood of $K$ values and the relationship between the number of E. japonica populations $(K)$ from 1 to 13, (d) Evanno table output, (e) bar plot representation, $K=4$. The purple and green represent the evergreen populations, and the blue and yellow represent the deciduous populations. 
Figure 1

Map showing the location of the sampled $E$. japonica populations

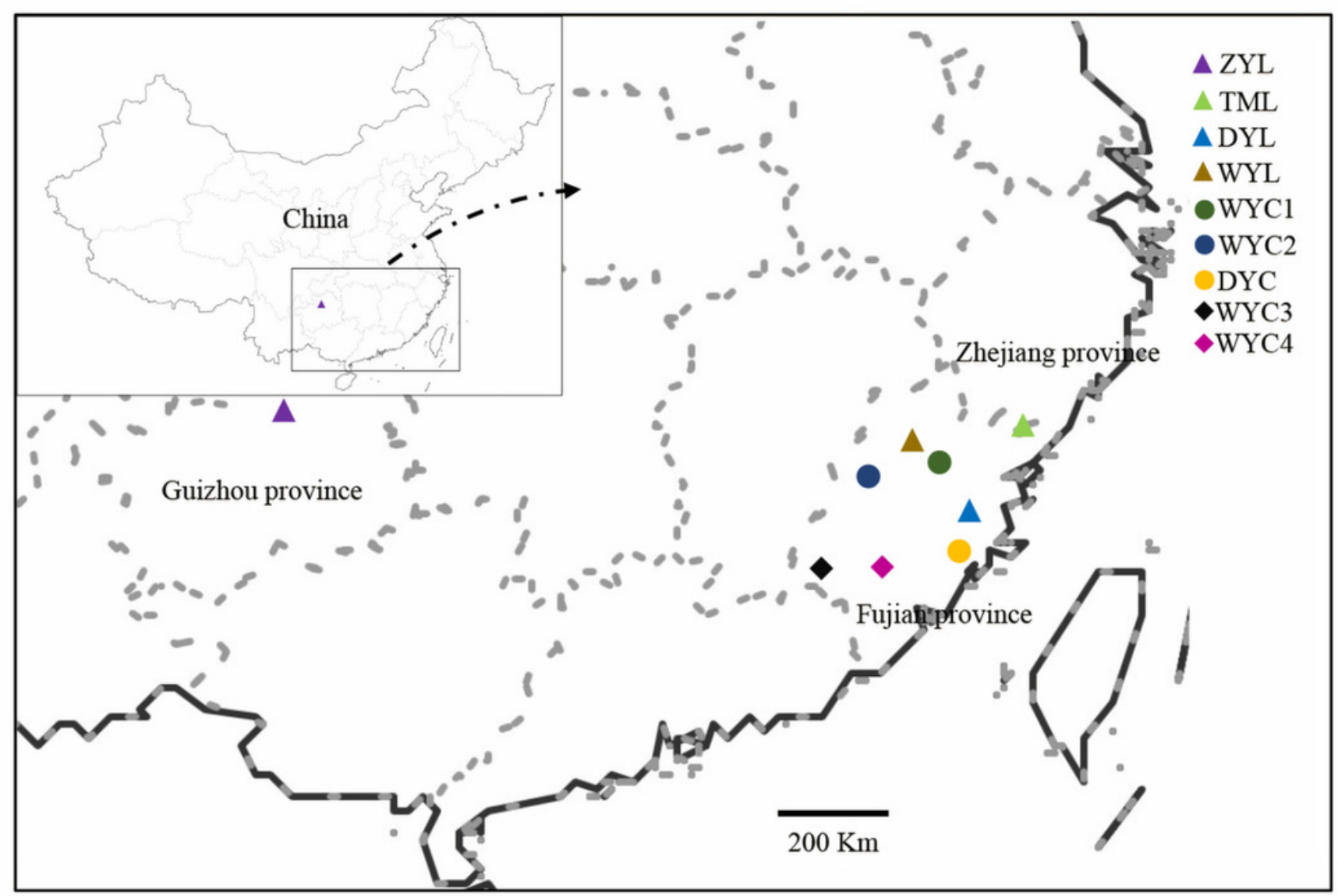


Figure 2

E. japonica population genetic structure
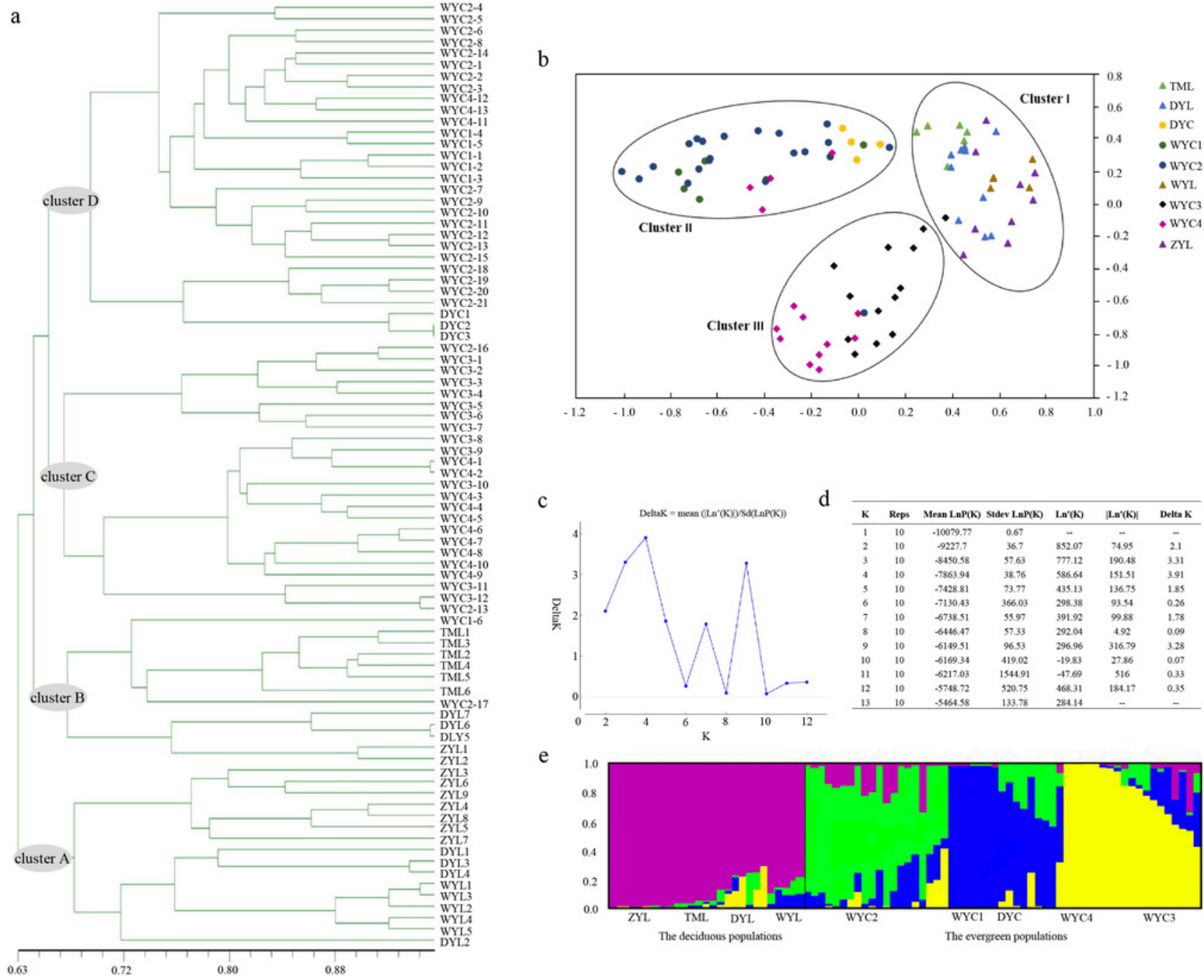

e

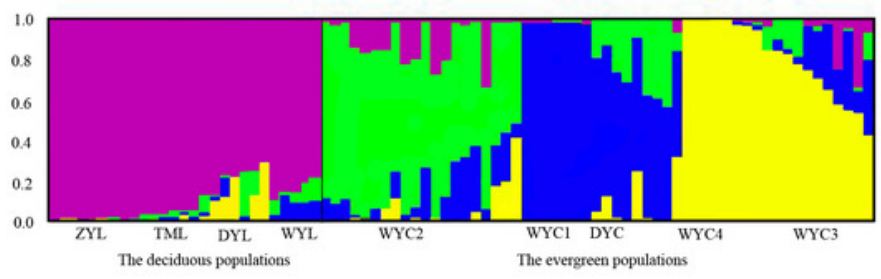




\section{Table 1 (on next page)}

The codes, locations, phenotypic character and habitat of populations of $E$. japonica 
Table 1. The codes, locations, phenotypic character and habitat of populations of $E$. japonica

\begin{tabular}{|c|c|c|c|c|c|c|c|}
\hline Location & population (size) & code & $\mathrm{E}$ & $\mathrm{N}$ & $\mathrm{A}(\mathrm{m})$ & Leaf characters & Fruit characters \\
\hline $\begin{array}{l}\text { Taimu Mountain, the border of } \\
\text { Fujian and Zhejiang Provinces }\end{array}$ & TML (6) & TML1 TML6 & $120^{\circ} 08^{\prime}$ & $27^{\circ} 23^{\prime}$ & 540 & Paper, margin sparsely serrulate & $\begin{array}{c}\text { pericarp softly leathery, red-brown with } \\
\text { irregular ribs }\end{array}$ \\
\hline \multirow{3}{*}{$\begin{array}{l}\text { Daiyun Mountain, Quanzhou } \\
\text { City, Fujian Province, }\end{array}$} & DYL (7) & DYL1 DYL7 & $118^{\circ} 53^{\prime}$ & $26^{\circ} 07^{\prime}$ & 946 & Paper, margin sparsely serrulate & $\begin{array}{c}\text { pericarp softly leathery, red-brown with } \\
\text { irregular ribs }\end{array}$ \\
\hline & DYC (3) & DYC1 DYC3 & $118^{\circ} 27^{\prime}$ & $25^{\circ} 42^{\prime}$ & 397 & Membrane, margin blunt serrations & Pericarp leathery, without irregular ribs \\
\hline & WYC1 (6) & WYC1-1 WYC1-6 & $118^{\circ} 18^{\prime}$ & $27^{\circ} 24^{\prime}$ & 181 & Membrane, margin blunt serrations & Pericarp leathery, without irregular ribs \\
\hline \multirow{2}{*}{\multicolumn{8}{|c|}{ Pericarp leathery, without irregular ribs }} \\
\hline & & & & & & & \\
\hline Sanming and Nanping Citys, & WYC3 (13) & WYC3-1 WYC3-13 & $116^{\circ} 48^{\prime}$ & $25^{\circ} 50^{\prime}$ & 340 & Membrane, margin blunt serrations & Pericarp leathery, without irregular ribs \\
\hline & WYC4 (13) & WYC4-1 WYC4-13 & $114^{\circ} 55^{\prime}$ & $25^{\circ} 23^{\prime}$ & 151 & Membrane, margin blunt serrations & Pericarp leathery, without irregular ribs \\
\hline & WYL (5) & WYL1 WYL5 & $118^{\circ} 02^{\prime}$ & $27^{\circ} 26^{\prime}$ & 508 & Paper, margin sparsely serrulate & $\begin{array}{c}\text { pericarp softly leathery, red-brown with } \\
\text { irregular ribs }\end{array}$ \\
\hline $\begin{array}{l}\text { Xishui National Reserve, } \\
\text { Zunyi, City, Guizhou Province }\end{array}$ & ZYL (9) & ZYL1 ZYL9 & $106^{\circ} 47^{\prime}$ & $28^{\circ} 49^{\prime}$ & 918 & Paper, margin sparsely serrulate & $\begin{array}{c}\text { pericarp softly leathery, red-brown with } \\
\text { irregular ribs }\end{array}$ \\
\hline
\end{tabular}

2 N: north latitude; E: west longitude; A: altitude (m). Population name ending in ' $\mathrm{L}$ ' represents the deciduous population, and population name ending in

3 ' $\mathrm{C}$ ' represents the evergreen population. 
Table 2 (on next page)

ISSR primer used for ISSR-PCR amplification and their amplification results 
Table 2. ISSR primer used for ISSR-PCR amplification and their amplification results

\begin{tabular}{cccccc}
\hline Primer & Sequence & \%GC & No. of bands & PIC & \multicolumn{2}{l}{$\mathrm{Nm}$} \\
\hline UBC807 & AGAGAGAGAGAGAG AGT & 47.1 & 12 & 0.57 & 1.4439 \\
UBC808 & AGAGAGAGAGAGAGAGC & 52.9 & 14 & 0.50 & 1.5361 \\
UBC809 & AGAGAGAGAGAGAGAGG & 52.9 & 10 & 0.62 & 1.8155 \\
UBC816 & CACACACACACACACAT & 53 & 10 & 0.61 & 2.3000 \\
UBC818 & CACACACACACACACAG & 52.9 & 9 & 0.55 & 0.8754 \\
UBC825 & ACACACACACACACACT & 47.1 & 9 & 0.58 & 3.3207 \\
UBC826 & ACACACACACACACACC & 52.9 & 9 & 0.66 & 0.5512 \\
UBC827 & ACACACACACACACACG & 52.9 & 10 & 0.52 & 1.5393 \\
UBC856 & ACACACACACACACACYA & 47.2 & 10 & 0.48 & 2.2742 \\
UBC861 & ACCACCACCACCACCACC & 66.7 & 12 & 0.50 & 1.6249 \\
UBC862 & AGCAGCAGCAGCAGCAGC & 66.7 & 11 & 0.61 & 1.2078 \\
UBC890 & VHVGTGTGTGTGTGTGT & 51.0 & 6 & 0.51 & 3.6068 \\
Mean & & & 10.17 & 0.56 & 0.7804 \\
\hline
\end{tabular}

2 
Table 3 (on next page)

Genetic diversity from E. japonica populations 
Table 3. Genetic diversity from $E$. japonica populations

\begin{tabular}{cccccccc}
\hline Population & $N a$ & $N e$ & $I$ & $H$ & $H e$ & $u H e$ & PPB (\%) \\
\hline TML & 1.3197 & 1.1735 & 0.1594 & 0.1046 & 0.1046 & 0.1141 & 31.97 \\
DYL & 1.4344 & 1.3857 & 0.3471 & 0.2301 & 0.2301 & 0.2422 & 67.21 \\
DYC & 1.2130 & 1.1244 & 0.1115 & 0.0738 & 0.0738 & 0.0843 & 21.31 \\
WYC1 & 1.2787 & 1.3483 & 0.2982 & 0.2003 & 0.2003 & 0.2185 & 55.74 \\
WYC2 & 1.7705 & 1.4390 & 0.3971 & 0.2607 & 0.2607 & 0.2670 & 86.07 \\
WYC3 & 1.4918 & 1.4276 & 0.3751 & 0.2512 & 0.2512 & 0.2612 & 70.49 \\
WYC4 & 1.5820 & 1.3610 & 0.3278 & 0.2141 & 0.2141 & 0.2220 & 72.13 \\
WYL & 0.7131 & 1.1448 & 0.1174 & 0.0803 & 0.0803 & 0.0892 & 20.49 \\
ZYL & 1.4426 & 1.4034 & 0.3548 & 0.2360 & 0.2360 & 0.2498 & 68.85 \\
The deciduous & 1.8443 & 1.4497 & 0.4133 & 0.2711 & 0.2676 & 0.1627 & 84.43 \\
The evergreen & 1.9754 & 1.4742 & 0.4455 & 0.2891 & 0.2854 & 0.2000 & 97.54 \\
Species level & 1.9918 & 1.4974 & 0.4630 & 0.3016 & 0.1834 & 0.1943 & 99.18 \\
\hline
\end{tabular}

2

3 


\section{Table 4(on next page)}

Fst value and the genetic similarity coefficient between populations 
Table 4. Fst value and the genetic similarity coefficient between populations

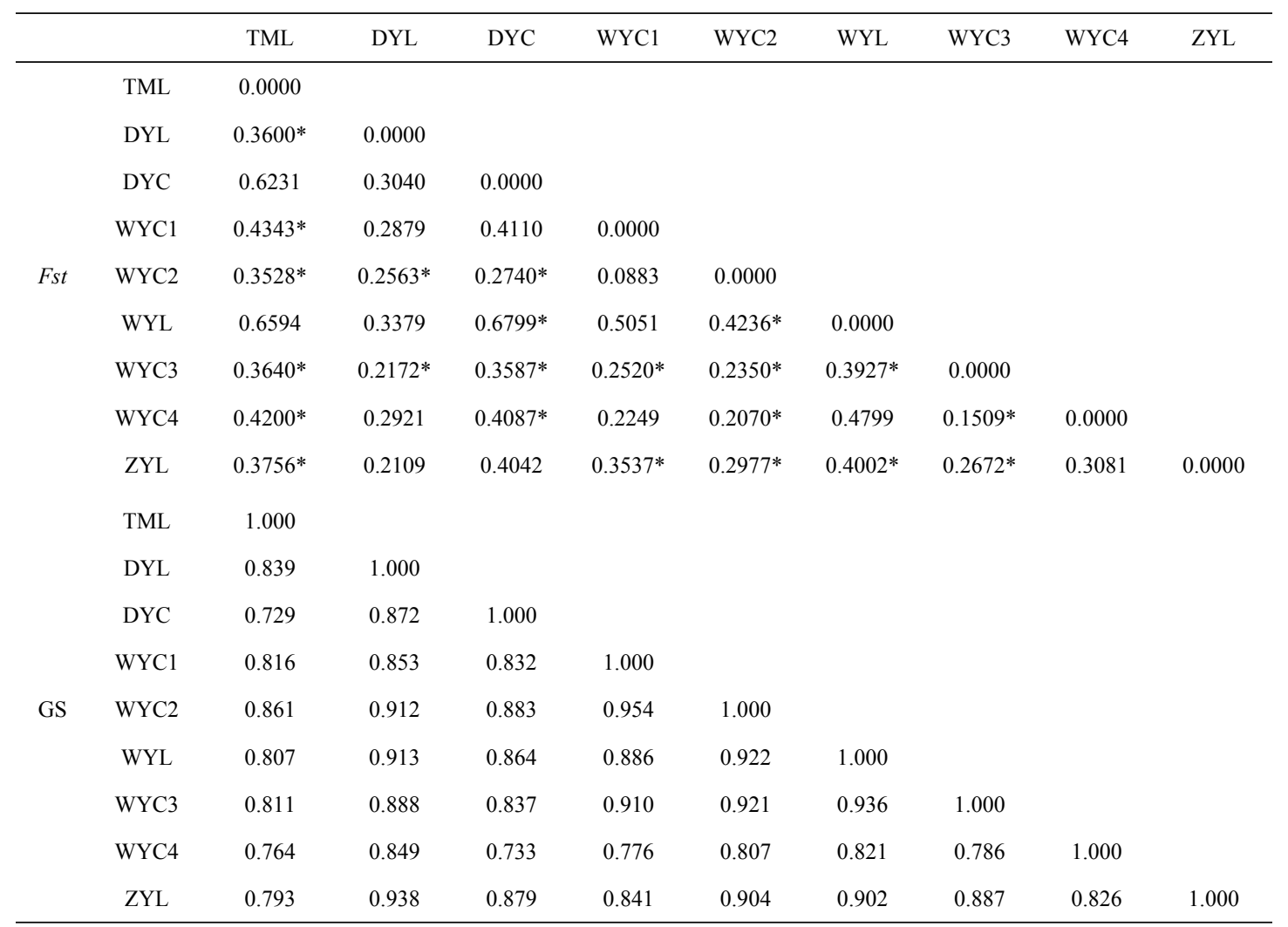

$2 *$ the difference is significant. GS means the Dice's genetic similarity coefficient. 


\section{Table 5 (on next page)}

The hierarchical analysis of molecular variance (AMOVA) examining differences among and within populations of $E$. japonica 
1 Table 5. The hierarchical analysis of molecular variance (AMOVA) examining differences among and

2 within populations of $E$. japonica

\begin{tabular}{ccccccc}
\hline & Source & df & SS & MS & Est. Var. & $\%$ \\
\hline \multirow{4}{*}{ All } & Among Populations & 8 & 605.564 & 75.696 & 6.455 & 30 \\
& Within Population & 79 & 1167.117 & 14.774 & 14.774 & 70 \\
& Total & 87 & 1772.682 & & 21.229 & 100 \\
\hline \multirow{4}{*}{ The Deciduous } & Among Populations & 3 & 205.99 & 68.641 & 7.538 & 36 \\
& Within Population & 26 & 351.844 & 13.532 & 13.532 & 64 \\
\hline \multirow{3}{*}{ The Evergreen } & Total & 29 & 557.767 & & 21.070 & 100 \\
\hline & Among Populations & 4 & 258.227 & 64.557 & 4.552 & 23 \\
& Within Population & 53 & 815.273 & 15.383 & 15.383 & 77 \\
\hline
\end{tabular}

3

4 